\title{
A PBW BASIS FOR LUSZTIG'S FORM OF UNTWISTED AFFINE QUANTUM GROUPS
}

\author{
FABIO GaVARini \\ Département de Mathématique - I.R.M.A. \\ Université "Louis Pasteur", Strasbourg
}

\begin{abstract}
Let $\hat{\mathfrak{g}}$ be an untwisted affine Kac-Moody algebra over the field $\mathbb{K}$, and let $U_{q}(\hat{\mathfrak{g}})$ be the associated quantum enveloping algebra; let $\mathfrak{U}_{q}(\hat{\mathfrak{g}})$ be the Lusztig's integer form of $U_{q}(\hat{\mathfrak{g}})$, generated by $q$-divided powers of Chevalley generators over a suitable subring $R$ of $\mathbb{K}(q)$. We prove a Poincaré-Birkhoff-Witt like theorem for $\mathfrak{U}_{q}(\hat{\mathfrak{g}})$, yielding a basis over $R$ made of ordered products of $q$-divided powers of suitable quantum root vectors.
\end{abstract}

\section{Introduction}

\author{
"Questa forma è duale \\ di un'altra già nota \\ che ha un suo teorem $P B W$. \\ Ed è subito base" \\ N. Barbecue, "Scholia"
}

Let $\hat{\mathfrak{g}}$ be an untwisted affine Kac-Moody algebra, and let $U_{q}(\hat{\mathfrak{g}})$ be the associated quantum enveloping algebra. In [Be1], [Be2], quantum root vectors are defined, and a basis of Poincaré-Birkhoff-Witt type for $U_{q}(\hat{\mathfrak{g}})$ is constructed, made of ordered monomials in the quantum root vectors.

Now let $\mathfrak{U}_{q}(\hat{\mathfrak{g}})$ be the Lusztig's integer form of $U_{q}(\hat{\mathfrak{g}})$, generated over $\mathbb{Z}\left[q, q^{-1}\right]$ by $q^{-}$ divided powers $E_{i}^{(n)}, F_{i}^{(m)}$; for technical reasons, we shall use a larger ground ring $R$. In this paper we find a PBW basis of $\mathfrak{U}_{q}(\hat{\mathfrak{g}})$ as an $R$-module, made of ordered products of $q$-divided powers of (suitable renormalizations of) quantum root vectors.

As a first step we reduce the problem to finding a basis for $\mathfrak{U}_{q}^{+}$(the positive part of $\left.\mathfrak{U}_{q}(\hat{\mathfrak{g}})\right)$. Second, we exploit the duality among PBW basis in $U_{q}^{+}$and in $U_{q}^{-}$- proved in [Da2] — to get from there our key result, namely finding a basis of $\mathfrak{U}_{q}^{+}$.

Such an approach is entirely different from the classical ones, to be found in [Ga] and [Mi]; on the other hand, the comparison with the classical setting is quite interesting: this

Revised version of June $17^{\text {th }} 1997$

1991 Mathematics Subject Classification, Primary 17B37, 81R50

Partially supported by a CNR post-doc fellowship 
is sketched in the last section, where also a second PBW theorem is proved and some further conjectures are presented.

\section{Acknowledgements}

The author wishes to thank J. Beck and V. Chari for explanations about their works, as well as I. Damiani and M. Rosso for many helpful discussions.

\section{$\S 1$ Notations}

1.1 The classical data. We shall adopt notations of [Da2], which we recall in this section (they follow $[\mathrm{Bo}]$ and $[\mathrm{Ka}]$ ).

Let $\mathbb{K}$ be any fixed algebraically closed field of characteristic zero. Let $\mathfrak{g}$ be a simple finite dimensional Lie algebra over $\mathbb{K}$, and consider the folllowing data.

$I_{0}=\{1, \ldots, n\}$, the set of vertices of the Dynkin diagram of $\mathfrak{g}$ (see [Da1] for the identification between $I_{0}$ and $\left.\{1, \ldots, n\}\right) ; \mathfrak{h}$ a Cartan subalgebra of $\mathfrak{g}$, generated by $\left\{h_{1}, \ldots, h_{n}\right\}=$ $\left\{h_{i} \mid i \in I_{0}\right\} ; \Phi_{0}=\Phi_{0,+} \cup\left(-\Phi_{0,+}\right) \subseteq \mathfrak{h}^{*}$ the root system of $\mathfrak{g}$, with $\Phi_{0,+}$ the set of positive roots, and $\Pi:=\left\{\alpha_{1}, \ldots, \alpha_{n}\right\}=\left\{\alpha_{i} \mid i \in I_{0}\right\}$ the set of simple roots; $Q_{0}:=\sum_{\alpha \in \Phi_{0}} \mathbb{Z} \alpha=\oplus_{i \in I_{0}} \mathbb{Z} \alpha_{i}$ the root lattice of $\mathfrak{g}$, and $Q_{0}^{\vee}:=\sum_{\alpha \in \Phi_{0}} \mathbb{Z} \alpha^{\vee}=\oplus_{i \in I_{0}} \mathbb{Z} \alpha_{i}^{\vee}$ the coroot lattice; $W_{0}$ the Weyl group of $\mathfrak{g}$. Finally, we fix a function $o: I_{0} \longrightarrow\{ \pm 1\}$ such that $a_{i j}<0 \Longrightarrow o(i) o(j)=-1$.

We denote $\hat{\mathfrak{g}}$ the untwisted affine Kac-Moody algebra associated to $\mathfrak{g}$ and we consider its loop-algebra like realization as

$$
\hat{\mathfrak{g}}=\mathfrak{g} \otimes_{\mathbb{K}} \mathbb{K}\left[t, t^{-1}\right] \oplus \mathbb{K} \cdot c \oplus \mathbb{K} \cdot \partial
$$

with the Lie bracket given as follows:

i) $c$ is central, i. e. $[c, x]=0 \forall x \in \hat{\mathfrak{g}}$;

ii) $\operatorname{ad}(\partial)=t \frac{d}{d t}$, i. e. $\left[\partial, x \otimes t^{m}\right]=m x \otimes t^{m} \forall x \in \mathfrak{g}, \forall m \in \mathbb{Z}$;

iii) $\left[x \otimes t^{r}, y \otimes t^{s}\right]=[x, y] \otimes t^{r+s}+\delta_{r,-s} r(x \mid y) c \forall x, y \in \mathfrak{g}, \forall r, s \in \mathbb{Z}$, where $(\cdot \mid \cdot)$ is the Killing form of $\mathfrak{g}$, normalized in such a way that $\left(h_{i}, h_{j}\right)=\frac{a_{i j}}{d_{j}}$.

For $\hat{\mathfrak{g}}$ we define: $I:=\{0,1, \ldots, n\} \supset I_{0}$ to be the set of vertices of the Dynkin diagram, and $I_{\infty}:=I \cup\{\infty\} ; A=\left(a_{i j}\right)_{i, j \in I}$ the (generalized) Cartan matrix and $D=\operatorname{diag}\left(d_{0}, d_{1}, \ldots, d_{n}\right)$ the diagonal matrix with relatively prime positive integral entries such that $D A$ is symmetric $\left(d_{0}=1\right) ; \hat{\mathfrak{h}}:=\mathfrak{h} \oplus \mathbb{K} \cdot c \oplus \mathbb{K} \cdot \partial(\subseteq \hat{\mathfrak{g}}) ; \Phi=\Phi_{+} \cup$ $\left(-\Phi_{+}\right)\left(\subset(\mathfrak{h} \oplus \mathbb{K} \cdot c)^{*} \subset \hat{\mathfrak{h}}^{*}\right)$ the root system, $\Phi_{+}=\Phi_{+}^{\mathrm{re}} \cup \Phi_{+}^{\text {im }}$ the set of positive roots, $\left\{\alpha_{0}, \alpha_{1}, \ldots, \alpha_{n}\right\}=\left\{\alpha_{i} \mid i \in I\right\}$ the set of simple roots, $\Phi_{+}^{\mathrm{im}}=\left\{m \delta \mid m \in \mathbb{N}_{+}\right\}$the set of imaginary positive roots (where $\delta=\sum_{i \in I} d_{i} \alpha_{i}=\theta+\alpha_{0}$ and $\theta$ is the longest positive root of $\mathfrak{g}), \Phi_{+}^{\mathrm{re}}=\Phi_{0,+} \cup\left\{\alpha+m \delta \mid \alpha \in \Phi_{0}, m>0\right\}$ the set of real positive roots. Then $\hat{\mathfrak{g}}$ has a decomposition into direct sum of $\hat{\mathfrak{h}}$ and root spaces $\hat{\mathfrak{g}}=\hat{\mathfrak{h}} \oplus\left(\oplus_{\alpha \in \Phi} \hat{\mathfrak{g}}_{\alpha}\right)$, and

$$
\operatorname{dim}_{\mathbb{C}}\left(\hat{\mathfrak{g}}_{\alpha}\right)=\left\{\begin{array}{ll}
1 & \text { if } \alpha \in \Phi^{\mathrm{re}} \\
\operatorname{dim}_{\mathbb{C}}(\mathfrak{h})=\#\left(I_{0}\right)=n & \text { if } \alpha \in \Phi^{\mathrm{im}}
\end{array} ;\right.
$$

therefore we define the set $\widetilde{\Phi}_{+}$of "positive roots with multiplicity" as $\widetilde{\Phi}_{+}:=\Phi_{+}^{\mathrm{re}} \cup \widetilde{\Phi}_{+}^{\mathrm{im}}$, where $\widetilde{\Phi}_{+}^{\mathrm{im}}:=\Phi_{+}^{\mathrm{im}} \times I_{0}$; then we denote $p: \widetilde{\Phi}_{+} \rightarrow \Phi_{+}$the natural projection map. Furthermore, we have: the root lattice (of $\hat{\mathfrak{g}}$ ) $Q=\sum_{\alpha \in \Phi} \mathbb{Z} \cdot \alpha=\oplus_{i \in I} \mathbb{Z} \cdot \alpha_{i}=\mathbb{Z} \cdot \alpha_{0} \oplus Q_{0}=$ 
$Q_{0} \oplus \mathbb{Z} \cdot \delta, Q_{\infty}:=Q \oplus \mathbb{Z} \cdot \alpha_{\infty}$, and the order relation $\leq$ on $Q_{\infty}$ given by $\alpha \leq \beta \Longleftrightarrow$ $\beta-\alpha \in Q_{+}$, with $Q_{+}:=\sum_{i \in I} \mathbb{N} \cdot \alpha_{i}$; the non-degenerate symmetric bilinear form on 
$Q_{\infty} \otimes_{\mathbb{Z}} \mathbb{R}$ given by $\left(\alpha_{i} \mid \alpha_{j}\right)=d_{i} a_{i j}(\forall i, j \in I),\left(\alpha_{\infty} \mid Q_{0} \oplus \mathbb{Z} \cdot \alpha_{\infty}\right)=0,\left(\alpha_{\infty} \mid \delta\right)=1$; the group $W=W_{0} \ltimes Q_{0}^{\vee}$, the subset of simple reflections $\left\{s_{0}, s_{1}, \ldots, s_{n}\right\}=\left\{s_{i} \mid i \in I\right\}(\subseteq$ $W$ ), and the length function $l: W \rightarrow \mathbb{N}$; the braid group $\mathcal{B}$ (associated to $W$ ), generated by $\left\{T_{0}, T_{1}, \ldots, T_{n}\right\}=\left\{T_{i} \mid i \in I\right\}$, and the section $T: W \rightarrow \mathcal{B}$ such that $T_{w}=T_{i_{1}} \cdots T_{i_{r}}$ for all $w=s_{i_{1}} \cdots s_{i_{r}} \in W$ with $l(w)=r$.

1.2 The quantum group $U_{q}(\hat{\mathfrak{g}})$. The quantized universal enveloping algebra $U_{q}(\hat{\mathfrak{g}})$ (cf. e.g. [Dr], [Lu2]) is the unital associative $\mathbb{K}(q)$-algebra with generators

$$
F_{1}, \ldots, F_{n}, K_{\nu}\left(\nu \in Q_{\infty}\right), E_{1}, \ldots, E_{n}
$$

and relations

$$
\begin{array}{cc}
K_{\mu} K_{\nu}=K_{\mu+\nu}=K_{\nu} K_{\mu}, \quad K_{0}=1 & \forall \mu, \nu \in Q_{\infty} \\
K_{\mu} E_{i}=q^{\left(\mu \mid \alpha_{i}\right)} E_{i} K_{\mu}, \quad K_{\mu} F_{i}=q^{-\left(\mu \mid \alpha_{i}\right)} F_{i} K_{\mu} & \forall \mu \in Q_{\infty}, \forall i \in I \\
E_{i} F_{j}-F_{j} E_{i}=\delta_{i j} \frac{K_{\alpha_{i}}-K_{-\alpha_{i}}}{q_{i}-q_{i}^{-1}} & \forall i, j \in I \\
\sum_{k=0}^{1-a_{i j}}(-1)^{k}\left[\begin{array}{c}
1-a_{i j} \\
k
\end{array}\right]_{q_{i}} E_{i}^{1-a_{i j}-k} E_{j} E_{i}^{k}=0 & \forall i \neq j \\
\sum_{k=0}^{1-a_{i j}}(-1)^{k}\left[\begin{array}{c}
1-a_{i j} \\
k
\end{array}\right]_{q_{i}} F_{i}^{1-a_{i j}-k} F_{j} F_{i}^{k}=0 & \forall i \neq j
\end{array}
$$

where

$$
\left[\begin{array}{c}
m \\
n
\end{array}\right]_{q}:=\frac{[m]_{q} !}{[m-n]_{q} ![n]_{q} !}, \quad[k]_{q} !:=\prod_{s=1}^{k}[s]_{q}, \quad[s]_{q}:=\frac{q^{s}-q^{-s}}{q-q^{-1}}
$$

for all $m, n, k, s \in \mathbb{N}_{+}, n \leq m$, with $[s]_{q},[k]_{q} !,\left[\begin{array}{c}m \\ n\end{array}\right]_{q} \in \mathbb{Z}\left[q, q^{-1}\right]$. For later use, we define

$$
q_{\alpha}:=\left\{\begin{array}{ll}
q^{\frac{(\alpha \mid \alpha)}{2}} & \forall \alpha \in \Phi_{+}^{\mathrm{re}} \\
q^{d_{i}} & \forall \alpha=(r \delta, i) \in \widetilde{\Phi}_{+}^{\mathrm{im}}
\end{array} \quad q_{i}:=q_{\alpha_{i}}=q^{d_{i}} \forall i \in I .\right.
$$

A Hopf algebra structure on $U_{q}(\hat{\mathfrak{g}})$ is defined by $\left(i=1, \ldots, n ; \mu \in Q_{\infty}\right)$

$$
\begin{array}{ccc}
\Delta\left(F_{i}\right):=F_{i} \otimes K_{-\alpha_{i}}+1 \otimes F_{i}, & S\left(F_{i}\right):=-F_{i} K_{\alpha_{i}}, & \epsilon\left(F_{i}\right):=0 \\
\Delta\left(K_{\mu}\right):=K_{\mu} \otimes K_{\mu}, \quad & S\left(K_{\mu}\right):=K_{-\mu}, & \epsilon\left(K_{\mu}\right):=1 \\
\Delta\left(E_{i}\right):=E_{i} \otimes 1+K_{\alpha_{i}} \otimes E_{i}, & S\left(E_{i}\right):=-K_{-\alpha_{i}} E_{i}, & \epsilon\left(E_{i}\right):=0
\end{array}
$$

Moreover, $U_{q}(\hat{\mathfrak{g}})$ has an algebra grading $U_{q}(\hat{\mathfrak{g}})=\oplus_{\eta \in Q} U_{q}(\hat{\mathfrak{g}})_{\eta}$.

We have a $\mathbb{K}$-linear antiinvolution $\Omega: U_{q}(\hat{\mathfrak{g}}) \rightarrow U_{q}(\hat{\mathfrak{g}})$ defined by

$$
\Omega(q):=q^{-1}, \quad \Omega\left(E_{i}\right):=F_{i}, \quad \Omega\left(K_{\nu}\right):=K_{-\nu}, \quad \Omega\left(E_{i}\right):=F_{i}, \quad \forall i \in I, \nu \in Q_{\infty}
$$

and a braid group action on $U_{q}(\hat{\mathfrak{g}})$ wich commutes with $\Omega$. 
Let $U_{q}^{+}, U_{q}^{0}, U_{q}^{-}$be the subalgebras of $U_{q}(\hat{\mathfrak{g}})$ respectively generated by $\left\{E_{i} \mid i \in I\right\}$, $\left\{K_{\nu} \mid \nu \in Q_{\infty}\right\},\left\{F_{i} \mid i \in I\right\}$; let also $U_{q}^{\geq}:=U_{q}^{+} \cdot U_{q}^{0}=U_{q}^{0} \cdot U_{q}^{+}, U_{q}^{\leq}:=U_{q}^{-} \cdot U_{q}^{0}=U_{q}^{0} \cdot U_{q}^{-}$, to be called quantum Borel (sub)algebras: these are Hopf subalgebras of $U_{q}(\hat{\mathfrak{g}})$. Finally, multiplication provides linear isomorphisms ("triangular decompositions")

$$
\begin{gathered}
U_{q}(\hat{\mathfrak{g}}) \cong U_{q}^{+} \otimes U_{q}^{0} \otimes U_{q}^{-} \cong U_{q}^{-} \otimes U_{q}^{0} \otimes U_{q}^{+} \\
U_{q}^{\geq} \cong U_{q}^{+} \otimes U_{q}^{0} \cong U_{q}^{0} \otimes U_{q}^{+}, \quad U_{q}^{\leq} \cong U_{q}^{0} \otimes U_{q}^{-} \cong U_{q}^{-} \otimes U_{q}^{0} .
\end{gathered}
$$

Remark: In the definition of $U_{q}(\hat{\mathfrak{g}})$ several choices for the "toral part" $U_{q}^{0}$ are possible, mainly depending on the choice of any lattice $M$ such that $Q \leq M \leq P, P$ being the weight lattice of $\mathfrak{g}(\mathrm{cf}$. for instance $[\mathrm{B}-\mathrm{K}])$. All what follows holds as well for every such choice, up to suitably adapting the statements involving the toral part.

1.3 The DRT pairing. Let $\left(U_{q}^{\leq}\right)^{\text {op }}$ denote the Hopf algebra with the same structure of $U_{q}^{\leq}$but for the coproduct, which is turned into the opposite one. Then a perfect (= non-degenerate) pairing of Hopf algebras $\pi: U_{q}^{\geq} \times\left(U_{q}^{\leq}\right)^{\text {op }} \longrightarrow \mathbb{K}(q)$ exists, defined by

$$
\pi\left(K_{\lambda}, K_{\mu}\right):=q^{-(\lambda \mid \mu)}, \quad \pi\left(K_{\lambda}, F_{i}\right):=0=: \pi\left(E_{i}, K_{\mu}\right), \quad \pi\left(E_{i}, F_{j}\right):=\frac{\delta_{i j}}{q_{i}^{-1}-q_{i}}
$$

for all $i, j \in I, \lambda, \mu \in Q_{\infty}$. This is a graded pairing, i. e. $\left.\pi\right|_{\left(U_{q}^{\geq}\right)_{\eta} \times\left(\left(U_{q}^{\leq}\right)^{\mathrm{op}}\right)_{\zeta}}=0$ for all $\eta, \zeta \in Q_{+}$such that $\eta+\zeta \neq 0$, and so $\left.\pi\right|_{\left(U_{q}^{\geq}\right)_{\eta} \times\left(\left(U_{q}^{\leq}\right)^{\mathrm{op}}\right)_{-\eta}}$ is non-degenerate $\eta \in Q_{+}$; finally, we have

$$
\pi\left(x K_{\lambda}, y\right)=\pi(x, y)=\pi\left(x, y K_{\mu}\right) \quad \forall x \in U_{q}^{\geq}, \forall y \in U_{q}^{\leq}, \forall \lambda, \mu \in Q_{\infty} .
$$

\section{$\S 2$ Quantum root vectors and PBW bases}

2.1 Ordering positive roots. In this section we sketch the construction of PBW bases, provided by Beck (cf. [Be1], [Be2]); here again we adopt the normalizations and notations of [Da2].

As in [Da2], $\S 2$, we can take a suitable function $\iota: \mathbb{Z} \rightarrow I$ (which we fix once and for all) such that the following holds: letting

$$
\beta_{k} \equiv \beta_{k}^{(\iota)}:= \begin{cases}s_{\iota(1)} s_{\iota(2)} \cdots s_{\iota(k-1)}\left(\alpha_{\iota(k)}\right) & \text { for all } k \geq 1 \\ s_{\iota(0)} s_{\iota(-1)} \cdots s_{\iota(k+1)}\left(\alpha_{\iota(k)}\right) & \text { for all } k \leq 0\end{cases}
$$

then $k \mapsto \beta_{k}$ defines a bijection of $\mathbb{Z}$ onto $\Phi_{+}^{\text {re }}$, so that $\left\{\beta_{k} \mid k \geq 1\right\}=\{r \delta-\alpha \mid r>$ $\left.0, \alpha \in \Phi_{0,+}\right\}$ and $\left\{\beta_{k} \mid k \leq 0\right\}=\left\{r \delta+\alpha \mid r \geq 0, \alpha \in \Phi_{0,+}\right\}$.

Then one defines a total ordering on $\widetilde{\Phi}_{+}$as follows:

$$
\begin{array}{r}
\beta_{1} \preceq \beta_{2} \preceq \beta_{3} \preceq \cdots \preceq \beta_{k-1} \preceq \beta_{k} \preceq \beta_{k+1} \preceq \cdots \\
\ldots((r+1) \delta, n) \preceq(r \delta, 1) \preceq(r \delta, 2) \cdots \preceq(2 \delta, n) \preceq(\delta, 1) \preceq(\delta, 2) \preceq \cdots \preceq(\delta, n) \preceq \cdots \\
\cdots \preceq \beta_{-(k+1)} \preceq \beta_{-k} \preceq \beta_{-(k-1)} \cdots \preceq \beta_{-2} \preceq \beta_{-1} \preceq \beta_{0}
\end{array}
$$


2.2 Quantum root vectors. Let $\iota$ (as in $\S 2.1$ ) be fixed. We define quantum root vectors as follows:

$$
\begin{gathered}
E_{\beta_{k}}^{(\iota)} \equiv E_{\beta_{k}}:=\left\{\begin{array}{lc}
T_{\iota(1)} T_{\iota(2)} \cdots T_{\iota(k-1)}\left(E_{\iota(k)}\right) & \text { for all } k \geq 1 \\
T_{\iota(0)}^{-1} T_{\iota(-1)}^{-1} \cdots T_{\iota(k+1)}^{-1}\left(E_{\iota(k)}\right) & \text { for all } k \leq 0
\end{array}\right. \\
\tilde{E}_{(r \delta, i)}^{(\iota)} \equiv \tilde{E}_{(r \delta, i)}:=q_{i}^{-2} E_{i} E_{r \delta-\alpha_{i}}-E_{r \delta-\alpha_{i}} E_{i} \quad \forall r>0, \forall i \in I_{0} \\
\left(q_{i}-q_{i}^{-1}\right) \sum_{r>0} E_{(r \delta, i)} \cdot \zeta^{r}:=\log \left(1-\left(q_{i}-q_{i}^{-1}\right) \sum_{s>0} \tilde{E}_{(s \delta, i)} \cdot \zeta^{r}\right) \\
\tilde{F}_{(r \delta, i)}:=\Omega\left(\tilde{E}_{(r \delta, i)}\right) \quad \forall(r \delta, i) \in \widetilde{\Phi}_{+}^{\mathrm{im}}, \quad F_{\alpha}:=\Omega\left(E_{\alpha}\right) \quad \forall \alpha \in \widetilde{\Phi}_{+}
\end{gathered}
$$

To be complete, we define also $\tilde{E}_{(0, i)}=E_{(0, i)}:=1=: \tilde{F}_{(0, i)}=F_{(0, i)}$ for all $i \in I_{0}$.

For later use, we define other imaginary root vectors by the recursive formulas

$$
\dot{E}_{(0 \delta, i)}:=1, \quad \dot{E}_{(r \delta, i)}:=q_{i}^{r} \frac{1}{[r]_{q_{i}}} \sum_{s=1}^{r} \tilde{E}_{(s \delta, i)} \dot{E}_{((r-s) \delta, i)} \quad \forall(r \delta, i) \in \widetilde{\Phi}_{+}^{\mathrm{im}}
$$

and $\dot{F}_{(r \delta, i)}:=\Omega\left(\dot{E}_{(r \delta, i)}\right)$; the relationship among the $\dot{E}_{(h \delta, i)}$ 's and the $E_{(k \delta, i)}$ 's is given by

$$
\dot{E}_{(0, i)}=1=E_{(0, i)}, \quad \dot{E}_{(r \delta, i)}=-\frac{1}{r} \sum_{s=1}^{r} q_{i}^{s} \frac{s}{[s]_{q_{i}}} E_{(s \delta, i)} \dot{E}_{((r-s) \delta, i)} \quad \forall(r \delta, i) \in \widetilde{\Phi}_{+}^{\mathrm{im}}
$$

(cf. [C-P], §3) and similarly with " $F "$ instead of " $E "$. Definitions give $E_{\beta} \in\left(U_{q}^{+}\right)_{\beta} \quad \forall \beta \in \Phi_{+}^{\mathrm{re}}, \quad \tilde{E}_{(r \delta, i)}, E_{(r \delta, i)}, \dot{E}_{(r \delta, i)} \in\left(U_{q}^{+}\right)_{r \delta} \quad \forall r \in \mathbb{N}, k \in N_{+}, i \in I_{0}$ and similarly for negative root vectors, with " $F "$ 's instead of " $E "$ 's. We shall not stick any more on the properties of such quantum root vectors, but for the following one, which will be useful later:

All quantum root vectors attached to imaginary roots commute with each other.

2.3 PBW bases. It is proved in $[\mathrm{Be} 2]$ that the set of ordered monomials in the root vectors $E_{\alpha}$ 's (according to the order $\preceq$ on $\Phi_{+}$defined in $\S 2.1$ ), namely the $\prod_{\alpha \in \Phi_{+}} E_{\alpha}^{n_{\alpha}}$ 's (where the $n_{\alpha} \in \mathbb{N}$ are almost all zero), is a $\mathbb{K}(q)$-basis of $U_{q}^{+}$; similarly, the set of ordered monomials in the root vectors $F_{\alpha}$ 's is a $\mathbb{K}(q)$-basis of $U_{q}^{-}$. Since clearly $\left\{K_{\alpha} \mid \alpha \in Q_{\infty}\right\}=$ $\left\{\prod_{i \in I_{\infty}} K_{i}^{l_{i}} \mid l_{i} \in \mathbb{Z} \forall i \in I_{\infty}\right\}$ is a $\mathbb{K}(q)$-basis of $U_{q}^{0}$, from triangular decompositions one concludes that the sets of ordered monomials $\prod_{\alpha \in \Phi_{+}} E_{\alpha}^{n_{\alpha}} \prod_{i \in I_{\infty}} K_{i}^{l_{i}} \prod_{\alpha \in \Phi_{+}} F_{\alpha}^{m_{\alpha}}$ or $\prod_{\alpha \in \Phi_{+}} F_{\alpha}^{m_{\alpha}} \prod_{i \in I_{\infty}} K_{i}^{l_{i}} \prod_{\alpha \in \Phi_{+}} E_{\alpha}^{n_{\alpha}}$ are $\mathbb{K}(q)$-bases of $U_{q}(\hat{\mathfrak{g}})$, and similarly for quantum Borel subalgebras.

2.4 Orthogonal bases. From [Da2] we know the values of the pairing of root vectors:

$$
\begin{gathered}
\pi\left(E_{\alpha}, F_{\beta}\right)=\frac{\delta_{\alpha, \beta}}{\left(q_{\alpha}^{-1}-q_{\alpha}\right)}, \pi\left(E_{\alpha}, F_{\gamma}\right)=0, \pi\left(E_{\gamma}, F_{\alpha}\right)=0 \quad \forall \alpha, \beta \in \Phi_{+}^{\mathrm{re}}, \gamma \in \widetilde{\Phi}_{+}^{\mathrm{im}} \\
\pi\left(E_{(r \delta, i)}, F_{(s \delta, j)}\right)=\delta_{r, s}(o(i) o(j))^{r} \frac{\left[r a_{i j}\right]_{q_{i}}}{r\left(q_{j}^{-1}-q_{j}\right)} \quad \forall(r, \delta, i),(s \delta, j) \in \widetilde{\Phi}_{+}^{\mathrm{im}} ;
\end{gathered}
$$

these formulas are the starting point to build up orthogonal bases of $U_{q}^{+}$and $U_{q}^{-}$. 
Lemma 2.5. For all $r \in \mathbb{N}_{+}$, let $V_{r}$, resp. $W_{r}$, be the $\mathbb{K}(q)$-vector space with basis $\left\{E_{(r \delta, i)} \mid i \in I_{0}\right\}$, resp. $\left\{F_{(r \delta, i)} \mid i \in I_{0}\right\}$, and let $\left\{x_{r, i} \mid i \in I_{0}\right\}$ and $\left\{y_{r, j} \mid j \in I_{0}\right\}$ be bases of $V_{r}$ and $W_{r}$ orthogonal of each other with respect to $\pi$, namely $\pi\left(x_{r, i}, y_{r, j}\right)=0$ for all $i \neq 0\left(i, j \in I_{0}\right)$. Then $\left\{\prod_{k \leq 0} E_{\beta_{k}}^{n_{k}} \cdot \prod_{r \in \mathbb{N}, i \in I_{0}} x_{r, i}^{n_{r, i}} \cdot \prod_{k>0} E_{\beta_{k}}^{n_{k}} \mid n_{k}, n_{r, i} \in \mathbb{N} \forall k, i\right\}$ and $\left\{\prod_{k \leq 0} F_{\beta_{k}}^{m_{k}} \cdot \prod_{r \in \mathbb{N}, j \in I_{0}} y_{s, j}^{m_{s, j}} \cdot \prod_{k>0} F_{\beta_{k}}^{m_{k}} \mid m_{k}, m_{s, j} \in \mathbb{N} \forall k, j\right\}$ are bases of $U_{q}^{+}$and $U_{q}^{-}$respectively which are orthogonal of each other. More precisely, we have

$$
\begin{aligned}
& \pi\left(\prod_{k \leq 0} E_{\beta_{k}}^{n_{k}} \cdot \prod_{r \in \mathbb{N}_{+}, i \in I_{0}} x_{r, i}^{n_{r, i}} \cdot \prod_{k>0} E_{\beta_{k}}^{n_{k}}, \prod_{h \leq 0} F_{\beta_{h}}^{m_{h}} \cdot \prod_{s \in \mathbb{N}_{+}, j \in I_{0}} y_{s, j}^{m_{s, j}} \cdot \prod_{h>0} F_{\beta_{h}}^{m_{h}}\right)= \\
& =\prod_{\alpha \in \Phi_{+}^{\mathrm{re}}} \delta_{n_{\alpha}, m_{\alpha}} q_{\alpha}^{\left(\begin{array}{c}
n_{\alpha} \\
2
\end{array}\right)} \frac{\left[n_{\alpha}\right]_{q_{\alpha}} !}{\left(q_{\alpha}^{-1}-q_{\alpha}\right)^{n_{\alpha}}} \cdot \prod_{r \in \mathbb{N}, i \in I_{0}} \delta_{n_{r, i}, m_{r, i}} n_{r, i} ! \pi\left(x_{r, i}, y_{r, i}\right)^{n_{r, i}}=
\end{aligned}
$$

Proof. This is just a variation of [Da2], Proposition 10.9, where a special choice of bases $\left\{x_{r, i} \mid i \in I_{0}\right\}$ and $\left\{y_{r, j} \mid j \in I_{0}\right\}$; but the sole point which is really necessary is just the orthogonality among such bases: once this is assumed, all the arguments used in [Da2], $\S 10$, to build up orthogonal (or even dual) bases go through as well without change.

\section{$\S 3$ Integer forms}

3.1 The ground ring. As our main goal in working with quantum groups is to specialize them at roots of 1 , we need integer forms of them defined over some ring in which $(q-\varepsilon)$ be not invertible, $\varepsilon$ being any fixed root of 1 . So let $\mathfrak{R}$ be the set of all roots of 1 (in $\mathbb{K}$ ) whose order is an odd number $\ell$ with $\ell=1$ or $\ell \geq 3$, and g.c.d. $(\ell, n+1)=1$ if $\mathfrak{g}$ is of type $A_{n}, \ell \notin 3 \mathbb{N}_{+}$if $\mathfrak{g}$ is of type $E_{6}$ or $G_{2}$; then set

$$
R_{\varepsilon}:=\mathbb{K}[q]_{(q-\varepsilon)}=\{f \in \mathbb{K}(q) \mid f \text { has no poles at } q=\varepsilon\} \forall \varepsilon \in \mathfrak{R}, \quad R:=\bigcap_{\varepsilon \in \mathfrak{R}} R_{\varepsilon} ;
$$

in particular, $\mathbb{Z}\left[q, q^{-1}\right] \subset R \subset R_{\varepsilon}$. Later on we shall need to inverte the determinants

$$
\Delta_{r}:=\operatorname{det}\left(\left(\left[a_{i j}\right]_{q_{i}^{r}}\right)_{i, j \in I_{0}}\right) \quad \forall r \in \mathbb{N}_{+} ;
$$

of course we have $\Delta_{r} \in \mathbb{Z}\left[q, q^{-1}\right]$, whence in particular $\Delta_{r} \in R \subset R_{\varepsilon}$. Here is the explicit description of the $\Delta_{r}$ 's, according to the type of $\mathfrak{g}$ :

$$
\begin{aligned}
& A_{n}:[n+1]_{q^{r}} \\
& B_{n}:[2]_{q^{(2 n-1) r}} \\
& C_{n}:[2]_{q^{(n+1) r}} \\
& D_{n}: \quad[2]_{q^{(n-1) r}} \cdot[2]_{q^{r}} \\
& E_{6}:[3]_{q^{r}} \cdot\left([2]_{q^{4 r}}-1\right) \\
& E_{7}: \quad[2]_{q^{r}} \cdot\left([2]_{q^{6 r}}-1\right) \\
& E_{8}:[2]_{q^{8 r}}+[2]_{q^{6 r}}-[2]_{q^{2 r}}-1 \\
& F_{4}:[2]_{q^{6 r}}-1 \\
& G_{2}:[3]_{q^{r}} \cdot\left([2]_{q^{10 r}}+[2]_{q^{8 r}}-[2]_{q^{2 r}}-1\right) .
\end{aligned}
$$

Note that, if $\varepsilon \in \mathfrak{R}$, then $\left.\Delta_{r}\right|_{q=\varepsilon} \neq 0$ for all $r \in \mathbb{N}_{+}$(by the very definition of $R_{\varepsilon}$ ); thus $\left.\Delta_{r}\right|_{q=\varepsilon}$ is invertible in each $R_{\varepsilon}$, whence also in $R$. 
3.2 Lusztig's integer form. Let us define the $q$-divided powers, namely

$$
F_{i}^{(\ell)}:=\frac{F_{i}^{\ell}}{[\ell]_{q_{i}} !}, \quad\left[\begin{array}{c}
K_{i} ; c \\
t
\end{array}\right]:=\prod_{s=1}^{t} \frac{q_{i}^{c-s+1} K_{i}-q_{i}^{-(c-s+1)} K_{i}^{-1}}{q_{i}^{s}-q_{i}^{-s}}, \quad E_{i}^{(m)}:=\frac{E_{i}^{m}}{[m]_{q_{i}} !}
$$

for all $i \in I, \ell, m, t \in \mathbb{N}, c \in \mathbb{Z}$. Then let $\mathfrak{U}_{q}(\hat{\mathfrak{g}})$ be the $R$-subalgebra of $U_{q}(\hat{\mathfrak{g}})$ generated by

$$
\left\{F_{i}^{(\ell)}, E_{i}^{(m)}, K_{i}^{ \pm 1} \mid i \in I, \ell, m \in \mathbb{N}\right\}
$$

this is a Hopf $R$-subalgebra of $U_{q}(\hat{\mathfrak{g}})(\mathrm{cf} .[\mathrm{Lu} 2])$. We set

$$
\mathfrak{U}_{q}^{-}:=\mathfrak{U}_{q}(\hat{\mathfrak{g}}) \cap U_{q}^{-}, \quad \mathfrak{U}_{q}^{0}:=\mathfrak{U}_{q}(\hat{\mathfrak{g}}) \cap U_{q}^{0}, \quad \mathfrak{U}_{q}^{+}:=\mathfrak{U}_{q}(\hat{\mathfrak{g}}) \cap U_{q}^{+} ;
$$

then (cf. [Lu2]) $\mathfrak{U}_{q}^{-}$, resp. $\mathfrak{U}_{q}^{0}$, resp. $\mathfrak{U}_{q}^{+}$, is the $R$-subalgebra of $U_{q}(\hat{\mathfrak{g}})$ generated by $\left\{F_{i}^{(\ell)} \mid\right.$ $i \in I, \ell \in \mathbb{N}\}$, resp. $\left\{\left[\begin{array}{c}K_{i} ; c \\ t\end{array}\right] K_{i}^{ \pm 1} \mid i \in I_{0}, t \in \mathbb{N}, c \in \mathbb{Z}\right\}$, resp. $\left\{E_{i}^{(m)} \mid i \in I, m \in \mathbb{N}\right\}$. Set

$$
\mathfrak{U}_{q}^{\leq}:=\mathfrak{U}_{q}(\hat{\mathfrak{g}}) \cap U_{q}^{\leq}, \quad \mathfrak{U}_{q}^{\geq}:=\mathfrak{U}_{q}(\hat{\mathfrak{g}}) \cap U_{q}^{\geq}
$$

then $\mathfrak{U}_{q}^{\leq}$, resp. $\mathfrak{U}_{q}^{\geq}$, is the $R$-subalgebra of $U_{q}(\hat{\mathfrak{g}})$ generated by $\mathfrak{U}_{q}^{-}$and $\mathfrak{U}_{q}^{0}$, resp. by $\mathfrak{U}_{q}^{0}$ and $\mathfrak{U}_{q}^{+}$; moreover, both $\mathfrak{U}_{q}^{\leq}$and $\mathfrak{U}_{\bar{q}}^{\geq}$are Hopf subalgebras (over $R$ ) of $\mathfrak{U}_{q}(\hat{\mathfrak{g}})$.

The triangular decompositions in $U_{q}(\hat{\mathfrak{g}})$ induce similar decompositions in $\mathfrak{U}_{q}(\hat{\mathfrak{g}})$, namely

$$
\begin{gathered}
\mathfrak{U}_{q}(\hat{\mathfrak{g}}) \cong \mathfrak{U}_{q}^{+} \otimes \mathfrak{U}_{q}^{0} \otimes \mathfrak{U}_{q}^{-} \cong \mathfrak{U}_{q}^{-} \otimes \mathfrak{U}_{q}^{0} \otimes \mathfrak{U}_{q}^{+} \\
\mathfrak{U}_{q}^{\geq} \cong \mathfrak{U}_{q}^{+} \otimes \mathfrak{U}_{q}^{0} \cong \mathfrak{U}_{q}^{0} \otimes \mathfrak{U}_{q}^{+}, \quad \mathfrak{U}_{q}^{\leq} \cong \mathfrak{U}_{q}^{0} \otimes \mathfrak{U}_{q}^{-} \cong \mathfrak{U}_{q}^{-} \otimes \mathfrak{U}_{q}^{0}
\end{gathered}
$$

which still are given by multiplication. Finally, the $\mathcal{B}$-action on $U_{q}(\hat{\mathfrak{g}})$ restricts on $\mathfrak{U}_{q}(\hat{\mathfrak{g}})$.

Remark: Some comments about the "toral part" $\mathfrak{U}_{q}^{0}$ of $\mathfrak{U}_{q}(\hat{\mathfrak{g}})$ are in order again. As in the finite case, several choices are possible: here we made the "minimal one", for we decide simply to take as $\mathfrak{U}_{q}(\hat{\mathfrak{g}})$ the smallest $R$-subalgebra containing the $q$-divided powers of Chevalley generators $E_{i}, F_{i}$ (for all $i$ ) and stable for the $\mathcal{B}$-action.

3.3 Beck-Kac's integer form. Define renormalized root vectors by

$$
\check{E}_{\alpha}:=\left(q_{\alpha}-q_{\alpha}^{-1}\right) E_{\alpha}, \quad \check{F}_{\alpha}:=\left(q_{\alpha}^{-1}-q_{\alpha}\right) F_{\alpha}, \quad \forall \alpha \in \widetilde{\Phi}_{+} ;
$$

(note that $\check{F}_{\alpha}=\Omega\left(\check{E}_{\alpha}\right)$ ), and let $\mathcal{U}_{q}(\hat{\mathfrak{g}})$ be the $R$-subalgebra of $U_{q}(\hat{\mathfrak{g}})$ generated by

$$
\left\{\check{F}_{\alpha}, K_{\mu}, \check{E}_{\alpha} \mid \alpha \in \Phi_{+}, \mu \in Q_{\infty}\right\}
$$

Then $($ cf. $[\mathrm{B}-\mathrm{K}]) \mathcal{U}_{q}(\hat{\mathfrak{g}})$ is a Hopf subalgebra of $U_{q}(\hat{\mathfrak{g}})$, with PBW basis over $R$

$$
\left\{\prod_{\alpha \in \Phi_{+}} \check{E}_{\alpha}^{n_{\alpha}} \cdot \prod_{i \in I_{\infty}} K_{i}^{l_{i}} \cdot \prod_{\alpha \in \Phi_{+}} \check{F}_{\alpha}^{m_{\alpha}} \mid l_{i} \in \mathbb{Z}, n_{\alpha}, m_{\alpha} \in \mathbb{N}, \forall i, \alpha ; \text { almost all } n_{\alpha}=m_{\alpha}=0\right\}
$$


(here again the monomials are ordered!); the latter is also a $\mathbb{K}(q)$-basis of $U_{q}(\hat{\mathfrak{g}})$, hence $\mathcal{U}_{q}(\hat{\mathfrak{g}})$ is an $R$-form of $U_{q}(\hat{\mathfrak{g}})$; furthermore, it is a Hopf subalgebra of $U_{q}(\hat{\mathfrak{g}})$. If we define

$$
\mathcal{U}_{q}^{-}:=\mathcal{U}_{q}(\hat{\mathfrak{g}}) \cap U_{q}^{-}, \quad \mathcal{U}_{q}^{0}:=\mathcal{U}_{q}(\hat{\mathfrak{g}}) \cap U_{q}^{0}, \quad \mathcal{U}_{q}^{+}:=\mathcal{U}_{q}(\hat{\mathfrak{g}}) \cap U_{q}^{+}
$$

then $\mathcal{U}_{q}^{-}$, resp. $\mathcal{U}_{q}^{0}$, resp. $\mathcal{U}_{q}^{+}$, is the $R$-subalgebra of $U_{q}(\hat{\mathfrak{g}})$ generated by $\left\{\check{F}_{\alpha} \mid \alpha \in \Phi_{+}\right\}$, resp. $\left\{K_{i}^{ \pm 1} \mid i \in I_{\infty}\right\}$, resp. $\left\{\check{E}_{\alpha} \mid \alpha \in \Phi_{+}\right\}$. Letting also

$$
\mathcal{U}_{q}^{\leq}:=\mathcal{U}_{q}(\hat{\mathfrak{g}}) \cap U_{q}^{\leq}, \quad \mathcal{U}_{q}^{\geq}:=\mathcal{U}_{q}(\hat{\mathfrak{g}}) \cap U_{q}^{\geq},
$$

we have that $\mathcal{U}_{q}^{\leq}$, resp. $\mathcal{U}_{q}^{\geq}$, is the $R$-subalgebra of $U_{q}(\hat{\mathfrak{g}})$ generated by $\mathcal{U}_{q}^{-}$and $\mathcal{U}_{q}^{0}$, resp. by $\mathcal{U}_{q}^{0}$ and $\mathcal{U}_{q}^{+}$; moreover, both $\mathcal{U}_{q}^{\leq}$and $\mathcal{U}_{q}^{\geq}$are Hopf subalgebras (over $R$ ) of $\mathcal{U}_{q}(\hat{\mathfrak{g}})$.

The triangular decompositions in $U_{q}(\hat{\mathfrak{g}})$ yield similar decompositions in $\mathcal{U}_{q}(\hat{\mathfrak{g}})$, namely

$$
\begin{gathered}
\mathcal{U}_{q}(\hat{\mathfrak{g}}) \cong \mathcal{U}_{q}^{+} \otimes \mathcal{U}_{q}^{0} \otimes \mathcal{U}_{q}^{-} \cong \mathcal{U}_{q}^{-} \otimes \mathcal{U}_{q}^{0} \otimes \mathcal{U}_{q}^{+} \\
\mathcal{U}_{q}^{\geq} \cong \mathcal{U}_{q}^{+} \otimes \mathcal{U}_{q}^{0} \cong \mathcal{U}_{q}^{0} \otimes \mathcal{U}_{q}^{+}, \quad \mathcal{U}_{q}^{\leq} \cong \mathcal{U}_{q}^{0} \otimes \mathcal{U}_{q}^{-} \cong \mathcal{U}_{q}^{-} \otimes \mathcal{U}_{q}^{0}
\end{gathered}
$$

where all the isomorphism are provided by multiplication.

It is clear now that PBW bases over $R$ also exist for the previous subalgebras of $\mathcal{U}_{q}(\hat{\mathfrak{g}})$ : for instance, the set of ordered monomials in the $\check{E}_{\alpha}^{\prime} s$ is a $R$-basis of $\mathcal{U}_{q}^{+}$; similarly, $\left\{\prod_{\alpha \in \Phi_{+}} \check{F}_{\alpha}^{m_{\alpha}} \cdot \prod_{i \in I_{\infty}} K_{i}^{t_{i}} \mid t_{i} \in \mathbb{Z}, m_{\alpha} \in \mathbb{N}\right.$, almost all $\left.m_{\alpha}=0\right\}$ is a $R$-basis of $\mathcal{U}_{q}^{\leq}$, etc.

Remark: The previous definitions and results are originally stated in [B-K] with $R_{\varepsilon}$ as ground ring; but since they hold for all $\varepsilon \in \mathfrak{R}$, one concludes that they hold over $R$ too.

\section{$\S 4$ The main PBW theorem}

4.1 $q$-divided powers. We extend the notion of $q$-divided powers to the root vectors attached to non-simple roots. To begin with, set

$$
\begin{array}{llll}
\hat{E}_{\alpha}:=E_{\alpha} & \forall \alpha \in \Phi_{+}^{\mathrm{re}}, & \hat{E}_{(r \delta, i)}:=\frac{r}{[r]_{q_{i}}} E_{(r \delta, i)} & \forall(r \delta, i) \in \widetilde{\Phi}_{+}^{\mathrm{im}} \\
\hat{F}_{\alpha}:=F_{\alpha} & \forall \alpha \in \Phi_{+}^{\mathrm{re}}, & \hat{F}_{(r \delta, i)}:=\frac{r}{[r]_{q_{i}}} F_{(r \delta, i)} & \forall(r \delta, i) \in \widetilde{\Phi}_{+}^{\mathrm{im}}
\end{array}
$$

note that $\hat{F}_{\alpha}=\Omega\left(\hat{E}_{\alpha}\right), \hat{E}_{\alpha}=\Omega\left(\hat{F}_{\alpha}\right)$. Then define $q$-divided powers of root vectors by

$$
\begin{aligned}
& E_{\alpha}^{(k)} \equiv \hat{E}_{\alpha}^{(k)}:=\frac{\hat{E}_{\alpha}^{k}}{[k]_{q_{\alpha}} !}, \quad \hat{E}_{(r \delta, i)}^{(k)}:=\frac{\hat{E}_{(r \delta, i)}^{k}}{k !} \\
& F_{\alpha}^{(k)} \equiv \hat{F}_{\alpha}^{(k)}:=\frac{\hat{F}_{\alpha}^{k}}{[k]_{q_{\alpha}} !}, \quad \hat{F}_{(r \delta, i)}^{(k)}:=\frac{\hat{F}_{(r \delta, i)}^{k}}{k !} \\
& \forall \alpha \in \Phi_{+}^{\mathrm{re}},(r \delta, i) \in \widetilde{\Phi}_{+}^{\mathrm{im}}, k \in \mathbb{N}
\end{aligned}
$$


Lemma 4.2. For all $\alpha \in \widetilde{\Phi}_{+}, k \in \mathbb{N}$, we have $\hat{E}_{\alpha}^{(k)} \in \mathfrak{U}_{q}^{+}, \hat{F}_{\alpha}^{(k)} \in \mathfrak{U}_{q}^{-}$.

Proof. It is enough to prove the claim for the $E$ 's.

If $\alpha \in \Phi_{+}^{\text {re }}$, then $\alpha=w\left(\alpha_{i}\right)$ for some $w \in W$ and some $i \in I$ : then $E_{\alpha}=T_{w}\left(E_{i}\right)$ by definition, so that $\hat{E}_{\alpha}^{(k)}=T_{w}\left(E_{i}^{(k)}\right)$ for all $k \in \mathbb{N}$. But $\mathfrak{U}_{q}(\hat{\mathfrak{g}})$ is $\mathcal{B}$-invariant (cf. [Lu2]), in particular $T_{w}\left(\mathfrak{U}_{q}(\hat{\mathfrak{g}})\right)=\mathfrak{U}_{q}(\hat{\mathfrak{g}})$, hence $\hat{E}_{\alpha}^{(k)} \in \mathfrak{U}_{q}(\hat{\mathfrak{g}})$, q.e.d.

If $\alpha \in \widetilde{\Phi}_{+}^{\mathrm{im}}$, say $\alpha=(r \delta, i)$, we proceed as follows. Consider the (imaginary) root vectors $\dot{E}_{(r \delta, i)}\left((r \delta, i) \in \widetilde{\Phi}_{+}^{\mathrm{im}}\right)$ defined in $\S 2.2$ : of course they lie in $U_{q}^{+}$; but even more, it is proved in $[\mathrm{C}-\mathrm{P}], \S 5$, that in fact it is

$$
\dot{E}_{(r \delta, i)} \in \mathfrak{U}_{q}^{+} \quad \forall(r \delta, i) \in \widetilde{\Phi}_{+}^{\mathrm{im}} .
$$

Now, the relationship between the $\dot{E}_{(h \delta, i)}$ 's and the $E_{(k \delta, i)}$ 's is given by (2.3); reversing that formula we get

$$
\frac{r}{[r]_{q_{i}}} E_{(r \delta, i)}=-r q_{i}^{-r} \dot{E}_{(r \delta, i)}-\sum_{h=1}^{r-1} q_{i}^{h-r} \frac{h}{[h]_{q_{i}}} E_{(h \delta, i)} \dot{E}_{((r-h) \delta, i)} \quad \forall(r \delta, i) \in \widetilde{\Phi}_{+}^{\mathrm{im}}
$$

which in terms of $\hat{E}_{(t \delta, i)}$ 's reads

$$
\hat{E}_{(r \delta, i)}=-r q_{i}^{-r} \dot{E}_{(r \delta, i)}-\sum_{h=1}^{r-1} q_{i}^{h-r} \hat{E}_{(h \delta, i)} \dot{E}_{((r-h) \delta, i)} \quad \forall(r \delta, i) \in \widetilde{\Phi}_{+}^{\mathrm{im}} ;
$$

this together with (4.3) gives us by induction

$$
\hat{E}_{(r \delta, i)} \in \mathfrak{U}_{q}^{+} \quad \forall r \in \mathbb{N}
$$

whence clearly $\hat{E}_{(r \delta, i)}^{(k)}:=\frac{1}{k !} \cdot \hat{E}_{(r \delta, i)}^{k} \in \mathfrak{U}_{q}^{+}$, q.e.d.

\section{Definition 4.3.}

$$
\begin{gathered}
\hat{\mathfrak{B}}_{+}:=\left\{\prod_{\alpha \in \Phi_{+}} \hat{E}_{\alpha}^{\left(n_{\alpha}\right)} \mid n_{\alpha} \in \mathbb{N} \forall i, \alpha ; \text { almost all } n_{\alpha}=0\right\} \\
\hat{\mathfrak{B}}_{0}:=\left\{\prod_{i \in I} K_{i}^{E n t\left(t_{i} / 2\right)}\left[\begin{array}{c}
K_{i} ; 0 \\
t_{i}
\end{array}\right]_{q_{i}} \mid t_{i} \in \mathbb{N} \forall i \in I\right\} \\
\hat{\mathfrak{B}}_{-}:=\left\{\prod_{\alpha \in \Phi_{+}} \hat{F}_{\alpha}^{\left(m_{\alpha}\right)} \mid m_{\alpha} \in \mathbb{N} \forall i, \alpha ; \text { almost all } m_{\alpha}=0\right\} .
\end{gathered}
$$

Then we define $\widehat{U}_{q}^{+}$, resp. $\widehat{U}_{q}^{0}$, resp. $\widehat{U}_{q}^{-}$, to be the $R$-submodule of $U_{q}^{+}$, resp. $U_{q}^{0}$, resp. $U_{q}^{-}$, spanned by $\hat{\mathfrak{B}}_{+}$, resp. $\hat{\mathfrak{B}}_{0}$, resp. $\hat{\mathfrak{B}}_{-}$.

Remark: By $\S 2.3$ it is clear that $\hat{\mathfrak{B}}_{\star}(\star \in\{+, 0,-\})$ is a $\mathbb{K}(q)$-basis of $U_{q}^{\star}$, hence $\widehat{U}_{q}^{\star}$ is a free $R$-module, with $\hat{\mathfrak{B}}_{\star}$ as an $R$-basis. 
Lemma 4.4. $\widehat{U}_{q}^{0}=\mathfrak{U}_{q}^{0}$; in other words, $\hat{\mathfrak{B}}_{0}$ is a basis of $\mathfrak{U}_{q}^{0}$ over $R$.

Proof. The proof is the same as in the finite case: see [Lu1], Theorem 6.7, and references therein.

Definition 4.5. Let $K$ be any field, let $A$ and $B$ be two $K$-algebras, and let $\pi: A \otimes B \longrightarrow$ $K$ be a $K$-bilinear pairing; assume $k$ is a subring of $K$ and $\widehat{A}$, resp. $\widehat{B}$, is an integer form of $A$, resp. $B$, over $k$. We say that $\widehat{A}$ is the $k$-dual of $\widehat{B}$ (with respect to $\pi$ ) if

$$
\widehat{A}=\{a \in A \mid \pi(a, \widehat{b}) \in k \forall \widehat{b} \in \widehat{B}\} .
$$

Proposition 4.6. $\widehat{U}_{q}^{+}$and $\mathcal{U}_{q}^{-}$, resp. $\widehat{U}_{q}^{-}$and $\mathcal{U}_{q}^{+}$, are $R$-dual of each other with respect to the DRT pairing.

Proof. Consider a positive imaginary root $r \delta, r \in \mathbb{N}_{+}$; from $\S 2.4$ and definitions we have

$$
\pi\left(\hat{E}_{(r \delta, i)}, \check{F}_{(r \delta, j)}\right)=(o(i) o(j))^{r} \frac{\left[r a_{i j}\right]_{q_{i}}}{[r]_{q_{i}}}=(o(i) o(j))^{r}\left[a_{i j}\right]_{q_{i}^{r}} \quad \forall i, j \in I_{0} .
$$

Consider the matrix

$$
M_{r}:=\left((o(i) o(j))^{r}\left[a_{i j}\right]_{q_{i}^{r}}\right)_{i, j \in I_{0}}
$$

we have $\operatorname{det}\left(M_{r}\right)= \pm \Delta_{r}$, thus $\operatorname{det}\left(M_{r}\right)$ is invertible in $R$, so the inverse matrix $M_{r}^{-1}=$ $\left(\mu_{i j}\right)_{i, j \in I_{0}}$ has all its entries in $R$; then define a new basis $\left\{\check{\mathcal{F}}_{(r \delta, j)} \mid j \in I_{0}\right\}$ of $W_{r}$ by

$$
\check{\mathcal{F}}_{(r \delta, i)}:=\sum_{j \in I_{0}} \mu_{j i} \check{F}_{(r \delta, j)} \quad \forall i \in I_{0} .
$$

By construction, we have now

$$
\pi\left(\hat{E}_{(r \delta, i)}, \check{\mathcal{F}}_{(r \delta, j)}\right)=\delta_{i j} \quad \forall i, j \in I_{0} .
$$

Moreover, since $\mu_{i j} \in R$ (for all $i, j$ ) and imaginary root vectors commute with each other, the set $\mathcal{B}_{-}$of ordered monomials

$$
\prod_{h \leq 0} \check{F}_{\beta_{h}}^{m_{h}} \cdot \prod_{s \in \mathbb{N}, j \in I_{0}} \check{\mathcal{F}}_{(s \delta, j)}^{m_{(s \delta, j)}} \cdot \prod_{h>0} \check{F}_{\beta_{h}}^{m_{h}}
$$

is again an $R$-basis of $\mathcal{U}_{q}^{-}$. The end of the story is that we can apply Lemma 2.5 , hence

$$
\begin{aligned}
& \pi\left(\prod_{k \leq 0} \hat{E}_{\beta_{k}}^{\left(n_{k}\right)} \cdot \prod_{r \in \mathbb{N}_{+}, i \in I_{0}} \hat{E}_{(r \delta, i)}^{\left(n_{(r \delta, i)}\right)} \cdot \prod_{k>0} \hat{E}_{\beta_{k}}^{\left(n_{k}\right)}, \prod_{h \leq 0} \check{F}_{\beta_{h}}^{m_{h}} \cdot \prod_{s \in \mathbb{N}_{+}, j \in I_{0}} \check{\mathcal{F}}_{(s \delta, j)}^{m_{(s \delta, j)}} \cdot \prod_{h>0} \check{F}_{\beta_{h}}^{m_{h}}\right)= \\
& =q_{\alpha}^{\sum_{\alpha \in \Phi_{+}^{\mathrm{re}}} d_{\alpha}\left(\begin{array}{c}
n_{\alpha} \\
2
\end{array}\right)} \prod_{\gamma \in \widetilde{\Phi}_{+}} \delta_{n_{\gamma}, m_{\gamma}} ;
\end{aligned}
$$


in particular, the last term is invertible in the ground ring $R$.

Now let $x \in U_{q}^{+}$: since $\hat{\mathfrak{B}}_{+}$is a basis of $U_{q}^{+}$over $\mathbb{K}(q)$, we have $x=\sum_{b \in \hat{\mathfrak{B}}_{+}} x_{b} b$ for some $x_{b} \in \mathbb{K}(q)$ (almost all zero).

Suppose $\pi(x, y) \in R$ for all $y \in \mathcal{U}_{q}^{-}$. Let $x_{b^{\prime}} \neq 0$ : by the previous analysis, there exists a (unique) monomial $\beta^{\prime}$ in the PBW basis $\mathcal{B}_{-}$of $\mathcal{U}_{q}^{-}$such that

$$
\pi\left(x, \beta^{\prime}\right)=\sum_{b \in \mathcal{B}_{+}} x_{b} \cdot \pi\left(b, \beta^{\prime}\right)=x_{b^{\prime}} q^{s}
$$

for some $s \in \mathbb{N}$; since $\pi\left(x, \beta^{\prime}\right) \in R$ by hypothesis, we have $x_{b^{\prime}} \in R$. We conclude that $x \in \widehat{U}_{q}^{+}$. Conversely, if $x_{b} \in R$ for all $b \in \hat{\mathfrak{B}}_{+}$, then for any monomial $\beta$ in $\mathcal{B}_{-}$we have

$$
\pi(x, \beta)=\sum_{b \in \mathcal{B}_{+}} x_{b} \cdot \pi(b, \beta)=x_{b^{\prime}} q^{s} \in R
$$

for a unique $b^{\prime} \in \mathcal{B}_{+}$and some $s \in \mathbb{N}$, whence $\pi(x, y) \in R$ for all $y \in \mathcal{U}_{q}^{-}$.

As $\hat{\mathfrak{B}}_{+}$is an $R$-basis of $\widehat{U}_{q}^{+}$, the proof is completed.

Theorem 4.7. $\mathfrak{U}_{q}^{ \pm}=\widehat{U}_{q}^{ \pm}$; in other words, $\hat{\mathfrak{B}}_{ \pm}$is a basis of $\mathfrak{U}_{q}^{ \pm}$over $R$.

Proof. By Lemma 4.2 and the definition of $\hat{\mathfrak{B}}_{+}$we get $\hat{\mathfrak{B}}_{+} \subseteq \mathfrak{U}_{q}^{+}$, whence $\widehat{U}_{q}^{+} \subseteq \mathfrak{U}_{q}^{+}$. On the other hand, thanks to Proposition 4.6 the converse inclusion will be proved if we show that $\pi\left(\mathfrak{U}_{q}^{+}, \mathcal{U}_{q}^{-}\right) \subseteq R$. The "-" case will follow applying $\Omega$.

Consider $E_{i}^{(k)}(i \in I, k \in \mathbb{N})$; when pairing $E_{i}^{(k)}$ with any monomial $\mathcal{F}$ in $\mathcal{B}_{-}$, the formula in Lemma 2.5 - cf. also the proof of Proposition 4.6 above - gives

$$
\pi\left(E_{i}^{(k)}, \mathcal{F}\right)=0 \quad \forall \mathcal{F} \neq \check{F}_{\alpha_{i}}^{(k)}, \quad \pi\left(E_{i}^{(k)}, \check{F}_{\alpha_{i}}^{k}\right)=q^{s}
$$

for some $s \in \mathbb{N}$; in particular, $\pi\left(E_{i}^{(k)}, \mathcal{F}\right) \in R$; therefore $E_{i}^{(k)} \in \widehat{U}_{q}^{+}$for all $i \in I, k \in \mathbb{N}$. But now remark that $\widehat{U}_{q}^{+}$is closed for multiplication, so that $\mathfrak{U}_{q}^{+} \subseteq \widehat{U}_{q}^{+}$, q.e.d. In fact, let $f, g \in \widehat{U}_{q}^{+}$; since $\pi$ is a Hopf pairing, we have

$$
\pi(f \cdot g, y)=\pi(f \otimes g, \Delta(y))
$$

for all $y \in \mathcal{U}_{q}^{-} \subseteq \mathcal{U}_{q}^{\leq}$; but $\mathcal{U}_{q}^{\leq}$is a Hopf $R$-algebra, hence $\Delta(y)=\sum_{(y)} y_{(1)} \otimes y_{(2)}$ with $y_{(1)}, y_{(2)} \in \mathcal{U}_{q}^{\leq}$, and so

$$
\pi(f \cdot g, y)=\pi(f \otimes g, \Delta(y))=\sum_{(y)} \pi\left(f, y_{(1)}\right) \cdot \pi\left(g, y_{(2)}\right) \in R, \text { q.e.d. }
$$

Remark: Notice that, if $\varepsilon \in \mathfrak{R}$ has order $\ell$ greater than 1 , then $\left.[r]_{q_{i}}\right|_{q=\varepsilon}=0$ for all $r \in \ell \mathbb{N}_{+} ;$similarly, $\left.\left[n_{\alpha}\right]_{q_{\alpha}} !\right|_{q=\varepsilon}=0$ for all $n_{\alpha} \geq \ell:$ this means that both $[r]_{q_{i}}$ and $\left[m_{\alpha}\right]_{q_{\alpha}}$ ! 
are not invertible in $R_{\varepsilon}$, hence - a fortiori - no more in $R$. Therefore the occurrence of coefficients $\left(\frac{1}{[r]_{q_{i}}}\right)^{n_{(r \delta, i)}}$ ("hidden inside" $\left.\hat{E}_{(r \delta, i)}^{\left(n_{(r \delta, i)}\right)}\right)$ and $\frac{1}{\left[n_{\alpha}\right]_{q_{\alpha}} !}$ ("hidden inside" $\left.\hat{E}_{\alpha}^{\left(n_{\alpha}\right)}\right)$, multiplying a monomial of Beck's basis for $U_{q}^{+}$, is really significant.

We conclude with our main result (a second PBW theorem will appear in $§ 5$ ):

First PBW Theorem 4.8. The sets of ordered monomials $\hat{\mathfrak{B}}_{+} \cdot \hat{\mathfrak{B}}_{0} \cdot \hat{\mathfrak{B}}_{-}$and $\hat{\mathfrak{B}}_{-} \cdot \hat{\mathfrak{B}}_{0} \cdot \hat{\mathfrak{B}}_{+}$ are bases of $\mathfrak{U}_{q}(\hat{\mathfrak{g}})$ over $R$.

Proof. Trivial from Lemma 4.4, Theorem 4.7, and triangular decomposition (cf. §3.2).

\section{$\S 5$ Generating series, the classical framework, and beyond}

5.1 Changing imaginary root vectors. Let consider sets of countably many variables $\mathbb{X}=\left\{X_{r}\right\}_{r \in \mathbb{N}}$, with $X_{0}=1$. Let such a set $\mathbb{X}$ be given: we define a new set $\mathbb{Y}=\left\{Y_{s}\right\}_{s \in \mathbb{N}}$ via the following relation among generating series:

$$
\sum_{s=0}^{\infty} Y_{s} \cdot \zeta^{s}=\exp \left(\sum_{r=1}^{\infty} X_{r} \cdot \zeta^{r}\right)
$$

(here $\zeta$ is any auxiliary symbol) notice this agree with $Y_{0}=1$. This is a variation on a classical theme: the $Y_{s}$ 's above are certain normalizations of the well-known complete Bell polynomials (cf. [Co], §3.3). We shall shortly denote any "change of variables" given by (5.1) with $\Psi: \mathbb{X} \succ \rightsquigarrow \mathbb{Y}$ or $\mathbb{X} \succ^{\Psi} \rightsquigarrow \mathbb{Y}$, and then write $\mathbb{Y}=\Psi(\mathbb{X})$. The converse change, to be denoted $\Phi: \mathbb{Y} \succ \rightsquigarrow \mathbb{X}$ or $\mathbb{Y} \succ^{\Phi} \leadsto \mathbb{X}$ or $\mathbb{X}=\Phi(\mathbb{Y})$, with $\Phi=\Psi^{-1}$, is given by

$$
\sum_{r=1}^{\infty} X_{r} \cdot \zeta^{r}=\log \left(\sum_{s=0}^{\infty} Y_{s} \cdot \zeta^{s}\right)
$$

Differentiating this identity (with respect to $\zeta$ ) and comparing the coefficients of $\zeta^{r}$ on both sides gives

$$
r Y_{r}=r X_{r}+\sum_{s=1}^{r-1} s X_{s} Y_{r-s}=\sum_{s=1}^{r} s X_{s} Y_{r-s} \quad \forall r \in \mathbb{N}_{+}
$$

which is a recursive rule to compute the $Y_{r}$ 's starting from the $X_{s}$ 's, i.e. to compute $\mathbb{Y}=\Psi(\mathbb{X})$. Reversing (5.3) gives the rule to compute $\mathbb{X}=\Phi(\mathbb{Y})$, namely

$$
r X_{r}=r Y_{r}-\sum_{s=1}^{r-1}(r-s) Y_{s} X_{r-s} \quad \forall r \in \mathbb{N}_{+} .
$$

Conversely, if $\mathbb{X}$ and $\mathbb{Y}$ verify (5.3) or (5.4), then $\mathbb{Y}=\Psi(\mathbb{X})$ and $\mathbb{X}=\Phi(\mathbb{Y})$. 
All this applies to our various sets of imaginary root vectors: for every $i \in I_{0}$ let

$$
\begin{gathered}
\tilde{\mathbb{E}}_{i}:=\left\{\tilde{E}_{(r \delta, i)}\right\}_{r \in \mathbb{N}}, \quad \mathbb{E}_{i}:=\left\{E_{(r \delta, i)}\right\}_{r \in \mathbb{N}}, \quad \dot{\mathbb{E}}_{i}:=\left\{\dot{E}_{(r \delta, i)}\right\}_{r \in \mathbb{N}}, \quad \hat{\mathbb{E}}_{i}:=\left\{\hat{E}_{(r \delta, i)}\right\}_{r \in \mathbb{N}} \\
-\left(q_{i}-q_{i}^{-1}\right) \tilde{\mathbb{E}}_{i}:=\left\{-\left(q_{i}-q_{i}^{-1}\right) \tilde{E}_{(r \delta, i)}\right\}_{r \in \mathbb{N}}, \quad\left(q_{i}-q_{i}^{-1}\right) \mathbb{E}_{i}:=\left\{\left(q_{i}-q_{i}^{-1}\right) E_{(r \delta, i)}\right\}_{r \in \mathbb{N}} \\
-\frac{q_{i}^{*}}{*} \hat{\mathbb{E}}_{i}:=\left\{-\frac{q_{i}^{r}}{r} \hat{E}_{(r \delta, i)}\right\}_{r \in \mathbb{N}} ;
\end{gathered}
$$

the relations in $\S 2.2$ tell us that: $-\left(q_{i}-q_{i}^{-1}\right) \tilde{\mathbb{E}}_{i} \overbrace{}^{\Phi} \nrightarrow\left(q_{i}-q_{i}^{-1}\right) \mathbb{E}_{i}$, by $(2.1)$, and simi-

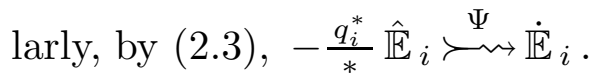

5.2 The classical setting. Recall that $\mathfrak{U}_{q}(\hat{\mathfrak{g}})$ is a quantization of $U(\hat{\mathfrak{g}})$, that is we have an isomorphism (of co-Poisson Hopf algebras)

$$
\mathfrak{U}_{q}(\hat{\mathfrak{g}}) /(q-1) \mathfrak{U}_{q}(\hat{\mathfrak{g}}) \cong U(\hat{\mathfrak{g}})
$$

The construction of the previous section are strictly related with some classical ones, inside $U(\hat{\mathfrak{g}})$. Namely, let $\hat{\mathfrak{g}}_{\mathbb{Z}}$ be the Lie subalgebra of $\hat{\mathfrak{g}}$ generated over $\mathbb{Z}$ by the Chevalley generators (assumed to be given) of $\hat{\mathfrak{g}}$; one can locate a Chevalley basis of $\hat{\mathfrak{g}}$, i.e. a $\mathbb{Z}$-basis of $\hat{\mathfrak{g}}_{\mathbb{Z}}$. Now let $U_{\mathbb{Z}}(\hat{\mathfrak{g}})$ be the $\mathbb{Z}$-subalgebra of $U(\hat{\mathfrak{g}})$ generated by the divided powers of the Chevalley generators: this is the classical counterpart of our $\mathfrak{U}_{q}(\hat{\mathfrak{g}})$, and the problem of finding a $\mathbb{Z}$-basis of it is the classical analogue of finding an $R$-basis of $\mathfrak{U}_{q}(\hat{\mathfrak{g}})$. The construction of a $\mathbb{Z}$-basis of $U_{\mathbb{Z}}(\hat{\mathfrak{g}})$ resembles that for the finite dimensional semisimple Lie algebras, but for one point, regarding imaginary root vectors.

Following [Mi], §4, pick out of the Chevalley basis of $\hat{\mathfrak{g}}$ the subset $\left\{\mathrm{e}_{\beta} \mid \beta \in \Phi_{+}^{\mathrm{re}}\right\}$ of root vectors attached to positive real roots and the subset $\left\{\tilde{\mathrm{e}}_{(r \delta, i)} \mid(r \delta, i) \in \widetilde{\Phi}_{+}^{\mathrm{im}}\right\}$ of root vectors attached to positive imaginary roots with multiplicity. For all $i \in I_{0}, k \in \mathbb{N}_{+}$, define a new set $\boldsymbol{\Lambda}_{i}^{\langle k\rangle}:=\left\{\Lambda_{[r ; i]}^{\langle k\rangle}\right\}_{r \in \mathbb{N}}$ by the change of variables

$$
\left\{\frac{1}{r} \tilde{\mathrm{e}}_{(r \cdot k \delta, i)}\right\}_{r \in \mathbb{N}} \stackrel{\Psi}{\hookrightarrow}^{\rightsquigarrow}\left\{\Lambda_{[r ; i]}^{\langle k\rangle}\right\}_{r \in \mathbb{N}}, \quad \text { in short } \quad \boldsymbol{\Lambda}_{i}^{\langle k\rangle}:=\Psi\left(\frac{1}{*} \tilde{\mathbf{e}}_{(k \delta)}\right) .
$$

Now fix an order on the Chevalley basis of $\hat{\mathfrak{g}}$, let $b^{(s)}:=\frac{b^{s}}{s !}$ denote usual divided powers, let $\hat{\mathfrak{g}}_{\mathbb{Z}}^{+}:=\hat{\mathfrak{g}}_{\mathbb{Z}} \bigcap \bigoplus_{\alpha \in \Phi_{+}} \hat{\mathfrak{g}}$, and set $U_{\mathbb{Z}}\left(\hat{\mathfrak{g}}^{+}\right):=U_{\mathbb{Z}}(\hat{\mathfrak{g}}) \cap U\left(\hat{\mathfrak{g}}^{+}\right)$(this is the classical counterpart

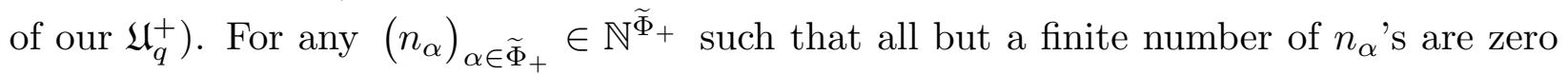
define the monomial $\mathcal{M}^{+}\left(\left(n_{\alpha}\right)_{\alpha \in \widetilde{\Phi}_{+}}\right)$to be the ordered product of the elements $\mathrm{e}_{\beta}^{\left(n_{\beta}\right)}$, $\Lambda_{\left[n_{(r \delta, i)} ; i\right]}^{\langle k\rangle}$ for all $\beta \in \Phi_{+}^{\mathrm{re}},(r \delta, i) \in \widetilde{\Phi}_{+}^{\mathrm{im}}, k \in \mathbb{N}_{+}$. The importance of the new sets of imaginary root vectors $\boldsymbol{\Lambda}_{i}^{\langle k\rangle}$ (for all $i \in I_{0}$ ) lies in the following result, due to Garland: 
Theorem $5.3([\mathbf{G a}],[\mathbf{M i}])$. The set of all monomials $\mathcal{M}^{+}\left(\left(n_{\alpha}\right)_{\alpha \in \widetilde{\Phi}_{+}}\right)$defined above is $a \mathbb{Z}$-basis of $U_{\mathbb{Z}}\left(\hat{\mathfrak{g}}^{+}\right)$.

5.4 Quantum versus classical imaginary root vectors. Comparison shows that Theorem 4.8 is not the direct $q$-analogue of its classical counterpart, Theorem 5.3.

First, Theorem 4.8 involves divided powers of imaginary root vectors, whereas Theorem 5.3 involves the $\Lambda_{\left[n_{(r \delta, i)} ; i\right]}^{\langle k\rangle}$ 's, which are not divided powers of imaginary root vectors; in this respect, the quantum result is somewhat nicer than the classical one.

Second, notice that in the isomorphism (5.5) one has

$$
\left.E_{\alpha}\right|_{q=1}=\mathrm{e}_{\alpha} \quad \forall \alpha \in \Phi_{+}^{\mathrm{re}},\left.\quad \tilde{E}_{(r \delta, i)}\right|_{q=1}=\tilde{\mathrm{e}}_{(r \delta, i)} \quad \forall(r \delta, i) \in \widetilde{\Phi}_{+}^{\mathrm{im}} ;
$$

now, the imaginary root vectors involved in Theorem 5.3 are built from those in the Cartan basis - the $\tilde{\mathrm{e}}_{(r \cdot k \delta, i)}$ 's - (up to normalization) through a change of variables "of type $\Psi$ "; on the other hand, in the quantum setting one starts from the $\tilde{E}_{(r \delta, i)}$ 's — which are the $q$-analogue of the $\tilde{\mathrm{e}}_{(r \cdot k \delta, i)}$ 's, thanks to (5.7) - but then the imaginary root vectors occurring in Theorem 4.8 are built from the $\tilde{E}_{(r \delta, i)}$ 's (up to normalizations, for $\hat{\mathbb{E}}_{i}$ is a renormalization of $\mathbb{E}_{i}$ ) through a change of variables "of type $\Phi$ "; in other words, starting from the same point the search for new suitable imaginary root vectors in the quantum case goes in opposite direction than in the classical setting.

Nevertheless, from Theorem 4.8 we can prove a second PBW Theorem, modeled on the classical one. To begin with, applying (5.4) to $-\left(q_{i}-q_{i}^{-1}\right) \tilde{\mathbb{E}}_{i} \stackrel{\Phi}{\hookrightarrow}^{\succ}\left(q_{i}-q_{i}^{-1}\right) \mathbb{E}_{i}$ gives

$$
r\left(q_{i}-q_{i}^{-1}\right) E_{(r \delta, i)}=-r\left(q_{i}-q_{i}^{-1}\right) \tilde{E}_{(r \delta, i)}+\sum_{s=1}^{r-1}(r-s)\left(q_{i}-q_{i}^{-1}\right)^{2} \tilde{E}_{(s \delta, i)} E_{((r-s) \delta, i)}
$$

which, dividing by $\left(q_{i}-q_{i}^{-1}\right)$, implies

$$
\left.E_{(r \delta, i)}\right|_{q=1}=-\left.\tilde{E}_{(r \delta, i)}\right|_{q=1}=-\tilde{\mathrm{e}}_{(r \delta, i)} \quad \forall(r \delta, i) \in \widetilde{\Phi}_{+}^{\mathrm{im}} ;
$$

furthermore, we have

$$
\left.\hat{E}_{(r \delta, i)}\right|_{q=1}=\left.E_{(r \delta, i)}\right|_{q=1}=-\tilde{\mathrm{e}}_{(r \delta, i)} \quad \forall(r \delta, i) \in \widetilde{\Phi}_{+}^{\mathrm{im}} .
$$

Now define a new set of imaginary root vectors $\dot{\mathbb{E}}_{i}^{[k]}=\left\{\dot{E}_{[r ; i]}^{[k]}\right\}_{r \in \mathbb{N}}\left(k \in \mathbb{N}_{+}\right)$by

$$
-\frac{q_{i}^{k \cdot *}}{*} \hat{\mathbb{E}}_{i}(k):=\left\{-\frac{q_{i}^{k \cdot r}}{r} \hat{E}_{(r \cdot k \delta, i)}\right\}_{r \in \mathbb{N}} \stackrel{\Psi}{\hookrightarrow}^{\Psi}\left\{\dot{E}_{[r ; i]}^{[k]}\right\}_{r \in \mathbb{N}}=: \dot{\mathbb{E}}_{i}^{[k]}
$$

(in particular, $\dot{\mathbb{E}}_{i}^{[1]}=\dot{\mathbb{E}}_{i}$, by definitions); explicitely, it is (for all $r, k \in \mathbb{N}_{+}, i \in I_{0}$ )

$$
\dot{E}_{[0 ; i]}^{[k]}:=1, \quad \dot{E}_{[r ; i]}^{[k]}:=-\frac{1}{r} \sum_{s=1}^{r} q_{i}^{k \cdot s} \frac{s \cdot k}{[s \cdot k]_{q_{i}}} E_{(s \cdot k \delta, i)} \dot{E}_{[(r-s) ; i]}^{[k]} \quad \forall(r \delta, i) \in \widetilde{\Phi}_{+}^{\mathrm{im}} .
$$


Since $\hat{\mathbb{E}}_{i} \subset \mathfrak{U}_{q}^{+}$, we have also $\hat{\mathbb{E}}_{i}(k) \subset \mathfrak{U}_{q}^{+}$, whence $\dot{\mathbb{E}}_{i}^{[k]} \subset \mathfrak{U}_{q}^{+} ;$moreover, from (5.6-10) we have $\left.\dot{\mathbb{E}}_{i}^{[k]}\right|_{q=1}=\boldsymbol{\Lambda}_{i}^{\langle k\rangle}$ : in other words, the $\dot{E}_{[r ; i]}^{[k]}$ 's are $q$-analogue of the $\Lambda_{[r ; i]}^{\langle k\rangle}$ 's.

Now we are ready for our second PBW theorem, which strictly mimicks the classical one.

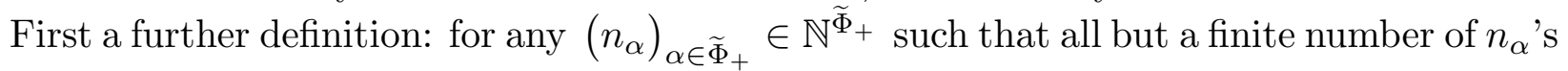
are zero define the monomial $\mathcal{M}_{q}^{+}\left(\left(n_{\alpha}\right)_{\alpha \in \widetilde{\Phi}_{+}}\right)$to be the ordered product - with respect to the ordered of $\widetilde{\Phi}_{+}$fixed in $\S 2.1$ - of the elements $E_{\beta}^{\left(n_{\beta}\right)}, \dot{E}_{\left[n_{(r \delta, i)} ; i\right]}^{[k]}$ for all $\beta \in \Phi_{+}^{\text {re }}$, $(r \delta, i) \in \widetilde{\Phi}_{+}^{\mathrm{im}}$; similarly define $\mathcal{M}_{q}^{-}\left(\left(n_{\alpha}\right)_{\alpha \in \widetilde{\Phi}_{+}}\right)$by means of negative root vectors (with $\dot{F}_{[r ; i]}^{[k]}:=\Omega\left(\dot{E}_{[r ; i]}^{[k]}\right)$ of course $)$, and call $\dot{\mathfrak{B}}_{ \pm}^{[*]}$ the set of all monomials $\mathcal{M}_{q}^{ \pm}\left(\left(n_{\alpha}\right)_{\alpha \in \widetilde{\Phi}_{+}}\right)$.

\section{Second PBW Theorem 5.5.}

(a) $\dot{\mathfrak{B}}_{+}^{[*]}$, resp. $\dot{\mathfrak{B}}_{-}^{[*]}$, is an $R$-basis of $\mathfrak{U}_{q}^{+}$, resp. $\mathfrak{U}_{q}^{-}$.

(b) The sets $\dot{\mathfrak{B}}_{+}^{[*]} \cdot \hat{\mathfrak{B}}_{0} \cdot \dot{\mathfrak{B}}_{-}^{[*]}$ and $\dot{\mathfrak{B}}_{-}^{[*]} \cdot \hat{\mathfrak{B}}_{0} \cdot \dot{\mathfrak{B}}_{+}^{[*]}$ are $R$-bases of $\mathfrak{U}_{q}(\hat{\mathfrak{g}})$.

Proof. Of course (b) follows from (a) and triangular decomposition.

As for (a), we proceed as follows. Inside $U\left(\hat{\mathfrak{g}}^{+}\right)$we have the basis provided by Theorem 5.3 and the usual basis of ordered monomials in the positive root vectors (of the given Chevalley basis): in particular, the first basis involve imaginary (positive) root vectors $\tilde{\mathrm{e}}_{(r \cdot k \delta, i)}$, whereas the second one involve the $\Lambda_{[r ; i]}^{\langle k\rangle}$, and the relationship among the two kinds of vectors is given by $\frac{1}{*} \tilde{\mathbf{e}}_{(k \delta)} \overbrace{}^{\Psi} \mathbf{\Lambda}_{i}^{\langle k\rangle}$. As they are both bases of $U\left(\hat{\mathfrak{g}}^{+}\right)$over $\mathbb{K}$, the matrix of basis change has entries in $\mathbb{K}$ (even more, in $\mathbb{Q}$ ).

Now, the situation in the quantum context is exactly the same; consider the set $\hat{\mathfrak{B}}_{+}^{\prime}$ defined like $\hat{\mathfrak{B}}_{+}$but for the fact that every $\hat{E}_{(z \delta, i)}$ is replaced by $q_{i}^{z} \hat{E}_{(z \delta, i)}$ : of course $\hat{\mathfrak{B}}_{+}^{\prime}$ is again an $R$-basis of $\mathfrak{U}_{q}^{+}$(thanks to Theorem 4.7 ). Then $\hat{\mathfrak{B}}_{+}^{\prime}$ and $\dot{\mathfrak{B}}_{+}^{[*]}$ differ only in the fact that they are built up in the same way but for the use of $\frac{1}{*}\left(-q_{i}^{k \cdot *} \hat{\mathbb{E}}_{i}(k)\right)$ in the first case

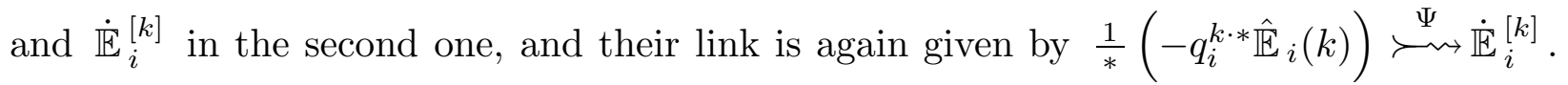
Therefore in force of the previous analysis these two sets must have the same $\mathbb{K}$-span (even more, the same $\mathbb{Q}$-span), whence the claim follows.

5.6 Integrality questions and beyond. Our constructions and results assume $R$ as ground ring; on the other hand, Lusztig's original definition of $\mathfrak{U}_{q}(\hat{\mathfrak{g}})$ takes $\mathbb{Z}\left[q, q^{-1}\right]$ as ground ring: thus the question arises of extending our results to such a setting.

Conjecturally, the definition of Beck-Kac's integer forms and their properties should hold as well over

(a) the smaller ring $\mathbb{K}\left[q, q^{-1}\right]$;

(b) the even smaller ring $\mathbb{Q}\left[q, q^{-1}\right]$;

(c) the smallest ring $\mathbb{Z}\left[q, q^{-1}\right]$.

If $W$ is anyone of the above rings, let $\mathcal{S}_{W}$ be the multiplicative part of $W$ generated by $\left\{\Delta_{r} \mid r \in \mathbb{N}_{+}\right\}$, and set $W_{\mathcal{S}}:=\mathcal{S}_{W}^{-1} W$.

In case $(a)$ or (b) would be true, it is immediate to check that all our arguments would go through as well if the ground ring is $\mathbb{K}\left[q, q^{-1}\right]_{\mathcal{S}}$ in case $(a)$ or if it is $\mathbb{Q}\left[q, q^{-1}\right]_{\mathcal{S}}$ in 
case (b). Case (c) is more tricky. First of all, we remark that an argument used in the proof of Lemma 4.2 can be refined: namely, formula (19) in [C-P] shows that in fact $\dot{E}_{(r \delta, i)}$ belongs to the Lusztig's form defined over $\mathbb{Z}\left[q, q^{-1}\right]$; therefore, the same steps in the cited proof tell the same is true for $\hat{E}_{(r \delta, i)}$ too, but this does no longer imply that the same holds for its divided powers $\hat{E}_{(r \delta, i)}^{(k)}(k \in \mathbb{N})$. In fact for sure Theorem 4.8 cannot hold over $\mathbb{Z}\left[q, q^{-1}\right]_{\mathcal{S}}$, because otherwise by specialization at $q=1$ it would imply - since $\left.E_{\beta}^{(k)}\right|_{q=1}=\mathrm{e}_{\beta}^{(k)},\left.\hat{E}_{\gamma}^{(k)}\right|_{q=1}=\tilde{\mathrm{e}}_{\gamma}^{(k)}$, for all $\beta \in \Phi_{+}^{\mathrm{re}}, \gamma \in \widetilde{\Phi}_{+}^{\mathrm{im}}$ - that the set of ordered products of divided powers of root vectors is a $\mathbb{Z}$-basis of $U_{\mathbb{Z}}\left(\hat{\mathfrak{g}}^{+}\right)$, which is false.

Things change for Theorem 5.5: its statement is really a $q$-analogue of the classical one, which relies on Theorem 5.3. This lead us to make the following

\section{Conjecture A.}

(a) $\dot{\mathfrak{B}}_{+}^{[*]}$, resp. $\dot{\mathfrak{B}}_{-}^{[*]}$, is a $\mathbb{Z}\left[q, q^{-1}\right]$-basis of the Lusztig's form of $U_{q}^{+}$, resp. $U_{q}^{-}$, defined over $\mathbb{Z}\left[q, q^{-1}\right]$.

(b) The sets $\dot{\mathfrak{B}}_{+}^{[*]} \cdot \hat{\mathfrak{B}}_{0} \cdot \dot{\mathfrak{B}}_{-}^{[*]}$ and $\dot{\mathfrak{B}}_{-}^{[*]} \cdot \hat{\mathfrak{B}}_{0} \cdot \dot{\mathfrak{B}}_{+}^{[*]}$ are $\mathbb{Z}\left[q, q^{-1}\right]$-bases of the Lusztig's form of $U_{q}(\hat{\mathfrak{g}})$ defined over $\mathbb{Z}\left[q, q^{-1}\right]$.

Furthermore, basing on the classical construction we can sketch an alternative approach.

Recall that in the classical setting to achieve a PBW theorem over $\mathbb{Z}$ one begins by the change of variables (5.6), i.e. $\left\{\frac{1}{r} \tilde{\mathrm{e}}_{(r . k \delta, i)}\right\}_{r \in \mathbb{N}} \stackrel{\Psi}{\longleftarrow}\left\{\Lambda_{[r ; i]}^{\langle k\rangle}\right\}_{r \in \mathbb{N}}$ : such a change is described either by a relation between generating series - namely any one of (5.1), (5.2) - or by a recursive formula - any one of (5.3), (5.4). In fact, in the original approach, to be found in [Ga], the $\Lambda_{[r ; i]}^{\langle k\rangle}$ 's are defined by means of a derivation operator and a multiplication operator (one for each $i$ ) acting on the $\tilde{\mathrm{e}}_{(r \cdot k \delta, i)}$ 's; and then one finds that the $\Lambda_{[r ; i]}^{\langle k\rangle}$ 's verify the cited recursive formulas. As a first attempt, one might try to "quantize" this method: exactly this is partially done in $[\mathrm{C}-\mathrm{P}]$. There the $q$-analogue of the $\Lambda_{[r ; i]}^{\langle k\rangle}$ 's, namely the $\dot{E}_{(r \delta, i)}$ 's ( $\equiv \dot{E}_{[r ; i]}^{[1]}$ ), are defined by means of $(2.2)$, which is nothing but a straight quantization of a recursive formula of type (5.3): after this the properties of the $\dot{E}_{[r ; i]}^{[1]}$ 's are obtained by working with a derivation operator and a multiplication operator which are the $q$-analogue of those used by Garland. To complete the task, one should generalize this by defining new vectors $\dot{E}_{[r ; i]}^{\langle k\rangle}$ for all $k \in \mathbb{N}_{+}$. First rewrite the right hand side formula in (2.2) as

$$
(r)_{q_{i}^{-2}} \dot{E}_{(r \delta, i)}:=\sum_{s=1}^{r}(s)_{q_{i}^{-2}}\left(\frac{q_{i}}{(s)_{q_{i}^{-2}}} \tilde{E}_{(s \delta, i)}\right) \dot{E}_{((r-s) \delta, i)} \quad \forall(r \delta, i) \in \widetilde{\Phi}_{+}^{\mathrm{im}}
$$

where we used the well-known notation $(z)_{q}:=\frac{q^{z}-1}{q-1}$; this gives $(2.2)$ the shape of a relation of type (5.3). Now generalize this defining $\left\{\dot{E}_{[r ; i]}^{\langle k\rangle}\right\}_{r \in \mathbb{N}}$ by means of

$$
(r)_{q_{i}^{-2}} \dot{E}_{[r ; i]}^{\langle k\rangle}:=\sum_{s=1}^{r}(s)_{q_{i}^{-2}}\left(\frac{q_{i}}{(s)_{q_{i}^{-2}}} \tilde{E}_{(s \cdot k \delta, i)}\right) \dot{E}_{[(r-s) ; i]}^{\langle k\rangle} \quad \forall i \in I_{0}, r, k \in \mathbb{N}_{+}
$$


i.e. $\quad \dot{E}_{[0 ; i]}^{\langle k\rangle}:=1, \dot{E}_{[r ; i]}^{\langle k\rangle}:=\frac{1}{(r)_{q_{i}^{-2}}} \sum_{s=1}^{r}(s)_{q_{i}^{-2}}\left(\frac{q_{i}}{(s)_{q_{i}^{-2}}} \tilde{E}_{(s \cdot k \delta, i)}\right) \dot{E}_{[(r-s) ; i]}^{\langle k\rangle} \quad \forall i \in I_{0}, r, k \in \mathbb{N}_{+}$

Since $\left.\tilde{E}_{(s \delta, i)}\right|_{q=1}=\tilde{\mathrm{e}}_{(s \delta, i)}$ and $\left.(r)_{q_{i}^{-2}}\right|_{q=1}=r$, definitions give $\left.\dot{E}_{[r ; i]}^{\langle k\rangle}\right|_{q=1}=\Lambda_{[r ; i]}^{\langle k\rangle}$, i.e. the $\dot{E}_{[r ; i]}^{\langle k\rangle}$ 's are $q$-analogue of the $\Lambda_{[r ; i]}^{\langle k\rangle}$ 's; the same is also true for the $\dot{E}_{[r ; i]}^{[k]}$ 's (cf. $\left.§ 5.4\right)$ and in fact $\dot{E}_{[r ; i]}^{\langle 1\rangle}=\dot{E}_{(r \delta, i)}=\dot{E}_{[r ; i]}^{[1]}$, but in general it is $\dot{E}_{[r ; i]}^{\langle k\rangle} \neq \dot{E}_{[r ; i]}^{[k]}$. Therefore, if we define the set $\dot{\mathfrak{B}}_{+}^{\langle *\rangle}$ just like the $\dot{\mathfrak{B}}_{+}^{[*]}$ but for using the $\dot{E}_{[r ; i]}^{\langle k\rangle}$ 's instead of the $\dot{E}_{[r ; i]}^{[k]}$ 's, and similarly for $\dot{\mathfrak{B}}_{-}^{\langle *\rangle}$, then the following statement should be the most proper $q$-analogue of the classical PBW theorem over $\mathbb{Z}$ (relying on Theorem 5.3):

\section{Conjecture B.}

(a) $\dot{\mathfrak{B}}_{+}^{\langle *\rangle}$, resp. $\dot{\mathfrak{B}}_{-}^{\langle *\rangle}$, is a $\mathbb{Z}\left[q, q^{-1}\right]$-basis of the Lusztig's form of $U_{q}^{+}$, resp. $U_{q}^{-}$, defined over $\mathbb{Z}\left[q, q^{-1}\right]$.

(b) The sets $\dot{\mathfrak{B}}_{+}^{\langle *\rangle} \cdot \hat{\mathfrak{B}}_{0} \cdot \dot{\mathfrak{B}}_{-}^{\langle *\rangle}$ and $\dot{\mathfrak{B}}_{-}^{\langle *\rangle} \cdot \hat{\mathfrak{B}}_{0} \cdot \dot{\mathfrak{B}}_{+}^{\langle *\rangle}$ are $\mathbb{Z}\left[q, q^{-1}\right]$-bases of the Lusztig's form of $U_{q}(\hat{\mathfrak{g}})$ defined over $\mathbb{Z}\left[q, q^{-1}\right]$.

A way to prove the previous conjecture might pass through the following alternative approach: quantizing the method in [Mi] instead of that in [Ga]. In fact in [Mi], one defines the $\Lambda_{[r ; i]}^{\langle k\rangle}$ 's via $(5.1)$ - or (5.2), it is equivalent - then establishes several relations involving generating series, exponentials and logarithms, and finally from these recovers Theorem 5.3 (and the most general result for the whole $U_{\mathbb{Z}}(\hat{\mathfrak{g}})$ ). So in order to quantize this track, one should try to directly quantize (5.1), (5.2), instead of (5.3), (5.4), then find $q$-analogues of the classical relations among generating series and the other functions involved in the "quantized (5.1), (5.2)", and from these achieve the claim of Conjecture B. Here we sketch some ideas which might lead to complete at least the first step.

Starting from (5.2), one gets (5.3) by

$$
\mathbb{X}(\zeta)=\log (\mathbb{Y}(\zeta)) \Longrightarrow \mathcal{D}(\mathbb{X}(\zeta))=\frac{\mathcal{D}(\mathbb{Y}(\zeta))}{\mathbb{Y}(\zeta)} \Longrightarrow \mathcal{D}(\mathbb{Y}(\zeta))=\mathcal{D}(\mathbb{X}(\zeta)) \cdot \mathbb{Y}
$$

(where $\mathbb{X}(\zeta):=\sum_{r=0}^{\infty} X_{r} \cdot \zeta^{r}, \mathbb{Y}(\zeta):=\sum_{s=0}^{\infty} Y_{s} \cdot \zeta^{s}$, and $\mathcal{D}$ denotes derivation with respect to $\zeta$ ) and comparison of coefficients of $\zeta^{t}(t \in \mathbb{N})$ on both sides. Then the coefficient $r$, resp. $s$, in the left, resp. right, hand side of (5.2) just arises from $\mathcal{D}\left(\zeta^{r}\right)$, resp. $\mathcal{D}\left(\zeta^{s}\right)$. Similarly, in (5.12) there are coefficients $(r)_{q_{i}^{-2}}$ and $(s)_{q_{i}^{-2}}$ which should come out in a similar way: thus, consider the skew-derivation $\mathcal{D}_{q}: A((\zeta)) \rightarrow A((\zeta))$ (here $A$ is any algebra containing the variables involved, namely the $X_{r}$ 's, the $Y_{s}$ 's, and so on) defined by

$$
\mathcal{D}_{q}: f(\zeta) \mapsto\left(\mathcal{D}_{q}(f)\right)(\zeta):=\frac{f(q \zeta)-f(\zeta)}{(q-1) \zeta} \quad \forall f(\zeta) \in A((\zeta))
$$

then we have $\mathcal{D}_{q}\left(\zeta^{n}\right)=(n)_{q} \zeta^{n-1}(n \in \mathbb{N})$, hence $\mathcal{D}_{q_{i}^{-2}}$ would fulfill our requirements. Second, a suitable function $\lambda o \gamma_{q}(q$-analogue of the logarithm) should be found such that 
$\mathcal{D}_{q_{i}^{-2}}\left(\lambda o \gamma_{q_{i}^{-2}}\left(\dot{\mathbb{E}}_{i}^{\langle k\rangle}(\zeta)\right)\right)=\frac{\mathcal{D}_{q_{i}^{-2}}\left(\dot{\mathbb{E}}_{i}^{\langle k\rangle}(\zeta)\right)}{\dot{\mathbb{E}}_{i}^{\langle k\rangle}(\zeta)}\left(\right.$ where $\left.\dot{\mathbb{E}}_{i}^{\langle k\rangle}(\zeta):=\sum_{r=0}^{\infty} \dot{E}_{[r ; i]}^{\langle k\rangle} \cdot \zeta^{r}\right) ;$ to this end, it should be useful the identity

$$
\dot{\mathbb{E}}_{i}^{\langle k\rangle}\left(q_{i}^{-2} \zeta\right)=\left(1-\left(q_{i}-q_{i}^{-1}\right) \sum_{r=1}^{\infty} \tilde{E}_{(r \cdot k, i)}\right) \cdot \dot{\mathbb{E}}_{i}^{\langle k\rangle}(\zeta)
$$

which arises from a reformulation of (5.12).

\section{REFERENCES}

[Be1] J. Beck, Braid group action and quantum affine algebras, Commun. Math. Phys. 165 (1994), $555-568$.

[Be2] J. Beck, Convex bases of PBW type for quantum affine algebras, Commun. Math. Phys. 165 (1994), 193-199.

[B-K] J. Beck, V. G. Kac, Finite dimensional representations of quantum affine algebras at roots of 1 , J. Amer. Math. Soc. 9 (1996), 391-423.

[Bo] N. Bourbaki, Groupes et algèbres de Lie, Chapitres 4-6, Hermann, Paris, 1968.

[Co] L. Comtet, Advanced Combinatorics, D. Reidel Publishing Company, Dordrecht-Holland/BostonU.S.A., 1974.

[C-P] V. Chari, A. Pressley, Quantum affine algebras at roots of unity, Preprint q-alg/9609031.

[Da1] I. Damiani, The highest coefficient of $H_{\eta}$ and the center at odd roots of 1 for untwisted affine quantum algebras, J. Algebra 186 (1996), 736-780.

[Da2] I. Damiani, La R-matrice pour les algèbres quantiques de type affine non tordu, Preprint.

[Dr] V. G. Drinfeld, Quantum groups, Proc. ICM Berkeley 1 (1986), 789-820.

[Ga] H. Garland, The arithmetic theory of loop algebras, J. Algebra 53 (1978), 480-551.

[Ka] V. G. Kac, Infinite Dimensional Lie Algebras, Birkhäuser, Boston, 1983.

[Lu1] G. Lusztig, Quantum groups at roots of 1, Geom. Dedicata 35 (1990), 89-113.

[Lu2] G. Lusztig, Introduction to quantum groups, Progress in Mathematics 110, Birkhäuser, Boston, 1993.

[Mi] D. Mitzman, Integral bases for affine Lie algebras and their universal enveloping algebras, Cont. Math. 40 (1985).

Département de Mathématique

Université "Louis Pasteur" - IRMA

7, Rue René Descartes

F-67084 Strasbourg - FRANCE

E-MAIL: GAVARINI@MATH.U-STRASBG.FR

GAVARINI@MAT.UNIROMA1.IT 